\title{
Increasing the Accuracy of Blood Hematocrit Measurement by Triplicate Wavelength Photoplethysmography Method
}

\author{
Mahdi Azarnoosh ${ }^{1^{*}}$, Hojjat Doostdar Noghabi ${ }^{2}$ \\ 1. Department of Biomedical Engineering, Mashhad Branch, Islamic Azad University, Mashhad, Iran. \\ 2. Department of Biomedical Engineering, Mashhad Branch, Islamic Azad University, Mashhad, Iran.
}

\section{Article Type:}

Original Article

\section{Article History:}

Received: 20 Sep. 2018

Revised: 1 Nov. 2018

Accepted: 5 Nov. 2018

\section{*Correspondence:}

Mahdi Azarnoosh

Assistant Professor,

Department of

Biomedical

Engineering,

Mashhad Branch,

Islamic Azad

University, Mashhad, Iran.

\section{Email:}

m_azarnoosh@mshdiau

.ac.ir

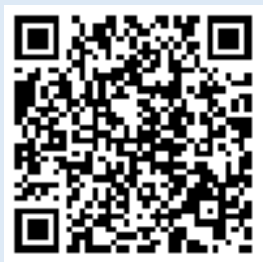

\begin{abstract}
Background and objectives: The importance of continuous monitoring and rapid and accurate informing of changes in blood components (e.g., percentage of hematocrit $[\mathrm{HCT}])$, especially in acute conditions, has motivated researchers to use non-invasive measurement methods. Therefore, this study aimed to evaluate the association between blood HCT and photoplethysmographic signal and the possibility of increasing the accuracy of its measurement by optical method at three specific wavelengths.
\end{abstract}

Methods: In this study, photoplethysmographic signals were recorded at three different wavelengths for 25 male and female subjects (mean age of $37.56 \pm 10.40$ years), who referred to the laboratory to assess their blood HCT percentage. We extracted the peak value of the signal after calibrating a special probe with a standard pulse oximetry system and applying the necessary preprocesses on the received signal. Eventually, in addition to the estimation of HCT level, we assessed the level of correlation between the optical method results and laboratory data applying Pearson's, Kendall's, and Spearman's correlation coefficients.

Results: Comparison of HCT measurement by the optical method and reference levels measured by standard laboratory technique in 25 subjects showed a mean error of $0.6 \pm 0.25 \%$. In addition, evaluation of the relationship between data of the percentage of laboratory HCT with mean of $43.43 \pm 3.43$ and data obtained by photoplethysmographic signals with mean of $43.31 \pm 3.27$ by three Pearson's, Kendall's, and Spearman's methods demonstrated that the data of the two techniques had a significant correlation of 0.949 , 0.860 , and 0.955 , respectively $(\mathrm{P}<0.01)$.

Conclusion: Given the high correlation of characteristics of the photoplethysmographic signal at three wavelengths with blood HCT and level of accuracy of our findings, the proposed method could be exploited for accurate, clean and cost-effective monitoring of HCT level.

Keywords: Hematocrit; Photoplethysmography; Near-infrared Spectrum

\section{Introduction}

Most medical diagnoses are based on analyzes of blood analytes. Hematocrit (HCT) is the ratio of the volume of red blood cells to the total volume of blood cells. The oxygen carrying capacity of blood is one of the necessary information provided by measuring

Copyright $@$ 2018, Jorjani Biomedicine Journal has published this work as an open access article under the terms of the Creative Commons Attribution License (http://creativecommons.org/licenses/by-nc/4.0/) which permits noncommercial uses of the work while it is properly cited. 
HCT. According to the literature, the blood HCT percentage in various groups has been reported at $39-50 \%, 35-45 \%$, and $30-40 \%$ for men, women, and small children and neonates, respectively. The most important causes of increased HCT levels include loss of body water, burn, diarrhea, and being in the postpartum period. High HCT levels are considered as a risk factor for myocardial infarction and stroke due to increased blood electrolytes. Moreover, increased HCT is one of the important factors in the formation of blood clots and increased risk of thrombosis.

Having a high HCT level endangers the health of patients during dialysis and open heart surgery, especially in patients with an artificial heart. On the other hand, decreased HCT is often the sign of anemia and suspected bleeding and can be due to bone marrow diseases, leukemia and malnutrition. In addition, any change in HCT will affect the level of safety of blood pump control. Therefore, the percentage of blood HCT should be evaluated during daily life as an index of control of various physiological conditions in order to reduce the risk factors for cardiovascular and other diseases. The standard methods for measuring HCT include a collection of blood samples in the capillary tube and separating red blood cells from the plasma by centrifugation. Eventually, by measuring the height of the red blood cells in the capillary tube to the total blood volume, the percentage of red blood cells that represents the percentage of HCT is measured (1).

Several studies have been performed in the area of HCT extraction by non-invasive methods for continuous monitoring. In this regard, two common methods for noninvasive measurement of HCT include optical and impedance techniques (2-6). Impedance method usually provides an incorrect answer when the level of protein and electrolytes are abnormal (e.g., in an unconscious state). On the other hand, the reported optical methods are typically based on different oximetric techniques, in which the HCT percentage is with the use of concentrations of deoxyhemoglobin and oxyhemoglobin of blood and is usually carried out with two or four wavelengths in the near-infrared spectrum (7-9). Since 1992, optical methods have often been applied in two wavelengths, the selective values of which were determined at 810 and $1300 \mathrm{~nm}$ (near-infrared spectrum) in the primary research (10).

Afterwards, the optical method was accepted for determining the concentration level of hemoglobin in different studies applying various wavelengths (11-14). In these studies, the researchers were able to determine the hemoglobin levels using two red and infrared wavelengths (15). However, the use of five wavelengths was also evaluated to increase measurement accuracy $(16,17)$. Moreover, the possibility of various artifacts has resulted in performing studies on their elimination applying hardware and software methods (1820). The importance and high use of the diagnosis of blood components using noninvasive optical methods due to its simplicity and cost-effectiveness, as well as higher accuracy and ability to measure several blood parameters simultaneously, compared to other methods, has led to the many studies and, consequently, numerous inventions in the field of detection of HCT and hemoglobin concentration (21-24).

In the present research, we proposed the use of photoplethysmography method at three wavelengths to provide an effective method for continuous monitoring of blood HCT levels. In addition, three wavelengths were 
applied for calibration and estimation of HCT level, where near-infrared spectrum was used jointly in two issues in order to optimize the design in terms of software and implementation, in addition to maintaining the system's accuracy and function. The results obtained from the optical method were compared with the data from the standard laboratory test so that the possibility of measuring the blood HCT levels with high accuracy in a non-invasive, inexpensive and simple way would be evaluated.

\section{Materials and Methods}

Signal recording: An invasive assay was performed applying a standard laboratory method by specialists and staff of Aria Hospital lab and Private Noor al-Mahdi Private Lab of Mashhad, Iran, exploiting a micro-hematocrit centrifuge device. Given the lack of considering the variables of gender, physiological conditions, or type of disease in HCT measurement, the subjects were selected from patients referring to the laboratories requiring HCT measurement as a part of their blood analysis. However, it was important to know that the proposed method of the present study did not interfere with their diagnostic and laboratory process. After explaining the research objectives and receiving consent, 25 patients (17 women and 8 men) in the age range of 25-68 years (mean age of $37.56 \pm 10.40$ ) were enrolled in the study. The photoplethysmography signal was recorded in three wavelengths of near-infrared $(850 \mathrm{~nm})$, red $(660 \mathrm{~nm})$, and green $(560 \mathrm{~nm})$ simultaneously by collecting blood samples from patients in the laboratory.

The data was recorded as a time multiplex in a channel and was sent to the computer after digitization. However, no analogue filter was applied in the recording probe. In addition, the signal recording was performed in the blood lab exploiting the PowerLab system. A schematic of the optical probes is shown in Figure 1. With regard to the application of two transmitters, they were placed in a circular form with a similar radius around the receptor, which was at the center of the circle. In the signal recording, the sampling frequency was $10 \mathrm{kHz}$. Given the fact that the researchers aimed to compare the HCT percentage obtained from the optical method directly with the extraction Reference data obtained from the standard method in the laboratory applying the invasive technique, no particular protocol was considered for signal recording and the status of the individuals at the time of signal recording was the same condition declared to the physician. During bloodletting, photoplethysmography signals were recorded at the mentioned wavelengths from the other hand of the subjects for 60 seconds. Figure (2) refers to the simultaneous recording of the photoplethysmography signals at several wavelengths during bloodletting in the laboratory. 


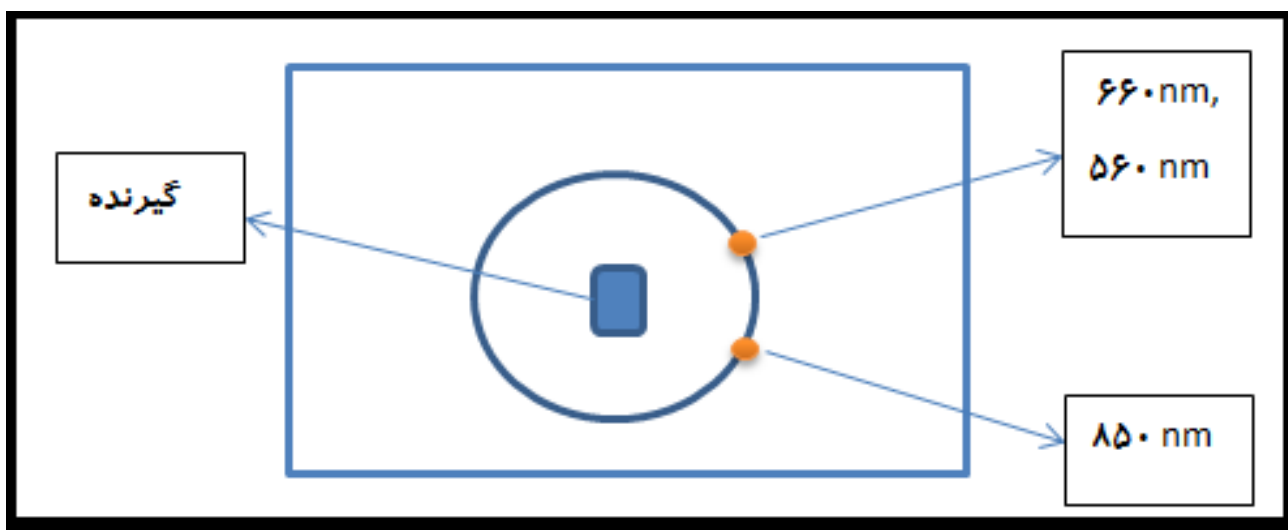

Figure 1: Photoplethysmographic signal recording probe at various wavelengths

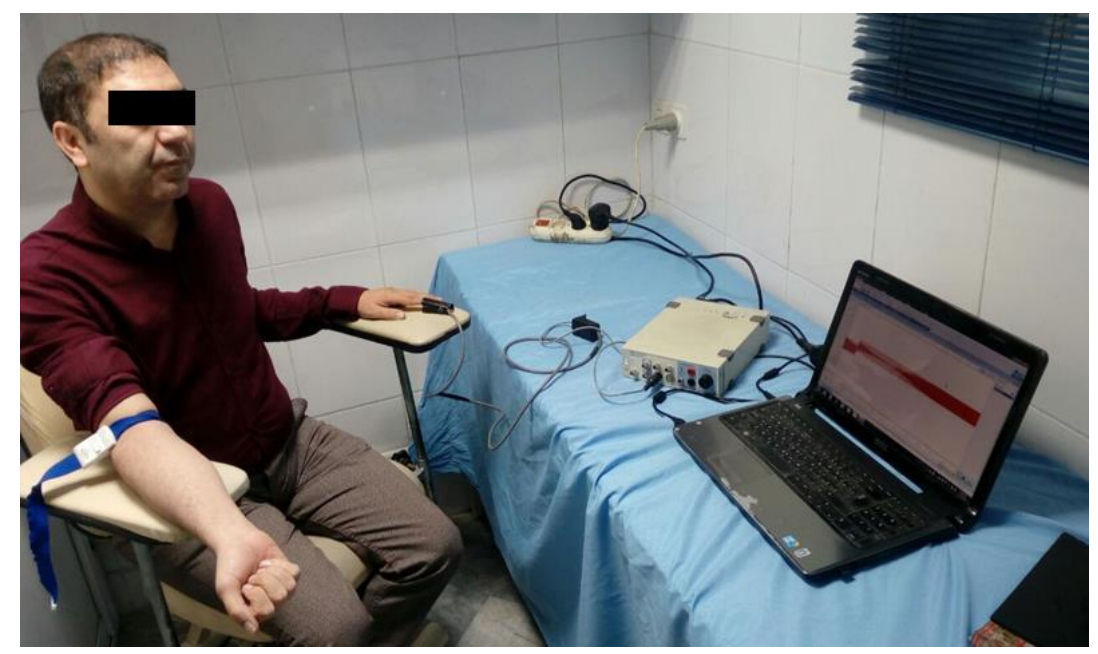

Figure 2: Recording of photoplethysmographic signal sample at three wavelengths by invasive bloodletting at laboratory

Preprocessing: Given the fact that the initial signal was in the form of multiplex time and had data related to each of the photoplethysmography signals at different wavelengths and at specific moments of times, isolation was required to be initially performed. However, no analogue filters were used on the probe in order to avoid any change in the control pulse shape. In this regard, a code is first defined and sent to detect the initiation moment the data. Recording the signal was succeeded by entering the data into MATLAB software, separating signals at various wavelengths, and eliminating the noise in the data digitally applying the mean filter. In addition, probe calibration was carried out by comparing the percentage of blood oxygen saturation to a standard pulse oximetry system (Novametrix Brand) for two wavelengths of 850 and 660 nm. Figure 3 shows the photoplethysmography signals at three wavelengths of an individual, which was recorded using the PowerLab data acquisition system and LabChart software.

HCT Estimation: Previously, two wavelengths of 880 and $570 \mathrm{~nm}$ were used to estimate the blood HCT. Considering that HCT is the ratio of red blood cell volume (Ve) to total blood volume ( $\mathrm{Vb})$, the following equation can be considered (25):

$$
H C T=\frac{V_{e}}{V_{b}}(1)
$$


On the other hand, according to the BeerLambert law for light intensity (I) and optical density (A), equations 2 and 3 are established, as follows:

$$
\begin{gathered}
I=I_{0} e^{-\varepsilon(\gamma) \propto d} \Rightarrow A^{\gamma}=\log \left(I_{0} / I\right)=\varepsilon(\gamma) c d \\
A_{t}=\sum_{i=1}^{n} \varepsilon_{i}(\gamma) c_{i} d_{i}
\end{gathered}
$$

In Equation $2, \varepsilon(\gamma)$ is the coefficient of light absorption at $\gamma$ wavelength, $\mathrm{c}$ is the concentration of the analyte in the tissue, $d$ is the length of path traveled by light from the transmitter to the receiver, ${ }^{I}$ is related to light intensity received in the receiver, and $I_{0}$ is related to the intensity of the transmitter's light, which can be estimated by measuring the transmitter and receiver flow. Equation (3) shows the concentration of $\mathrm{n}$ different materials can be determined using BeerLambert law, if the absorption coefficient of that material in $\mathrm{n}$ different wavelengths is known. However, it is impossible to precisely determine the path traveled by light in the tissue from the transmitter to receiver and it must be estimated based on experimental tests and techniques such as Monte Carlo method (26). In this study, wavelengths of 950, 850, 740, 660, and $560 \mathrm{~nm}$ were used, and the simulation and test results indicated that two wavelengths of 850 and $560 \mathrm{~nm}$ could be applied as a useful tool to estimate blood HCT. Accordingly, Equation (4) is introduced to estimate HCT levels:

$$
H C T_{E}=\frac{C^{560} \times A^{560}}{C^{560} \times A^{560}+C^{850} \times A^{850}}
$$

In Equations 4, C560 and C850 are calibration coefficients, which can be determined empirically and based on simulation and test results and by comparison with actual data. In addition, the amount of $\mathrm{A}$ can be calculated according to the light intensity of the transmitter and receiver by equation (2).

Result assessment method: this was an analytical observational study, the goal of which was assessing the relationship between the results of the obtained from the proposed optical method and the standard laboratory method. Therefore, in addition to evaluating the difference between the data obtained from the reference laboratory values, the correlation between the percentage of estimated HCT by the proposed optical method with the data reported from blood laboratory was assessed. In order to determine the sample size with regard to the type of correlational statistical studies and based on the maximum acceptable errors in each of these tests, the calculations were performed according to the relationships, which led to the selection of 25 subjects (27).

To this end, we first used the Pearson correlation coefficient, which is one of the 
most popular methods for determining the extent and the relationship between two variables. This condition was also evaluated considering the necessity of the normal distribution of the data. In addition, Kendall's and Spearman's rank correlation coefficient was used to evaluate the level of correlation of changes in two variables after their sorting

\section{Results}

The results of the blood HCT test in the laboratory based on the decrease in the rate of all subjects in the descending order are shown in Figure 4. Laboratory data related to the in descending order. These tests are nonparametric and there is no need for normality of the data. Data analysis was performed in SPSS version 21, and engineering analyzes, separation of signals, and displaying results were carried out using Matlab Software 2016.

blood HCT percentage in terms of normal distribution was assessed by KolmogorovSmirnov test, results of which showed that there was no reason to reject the null hypothesis based on the normal distribution of data $(\mathrm{P}<0.05)$.

A)

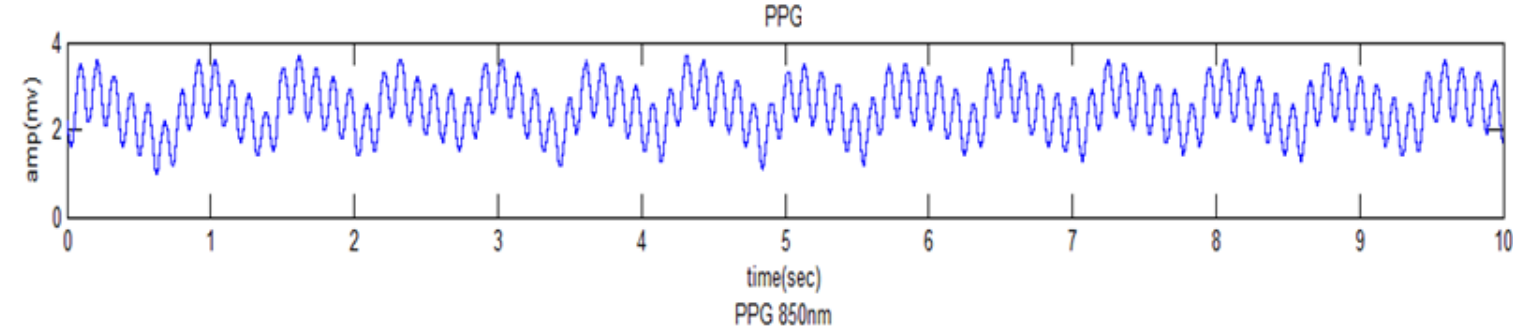

B)

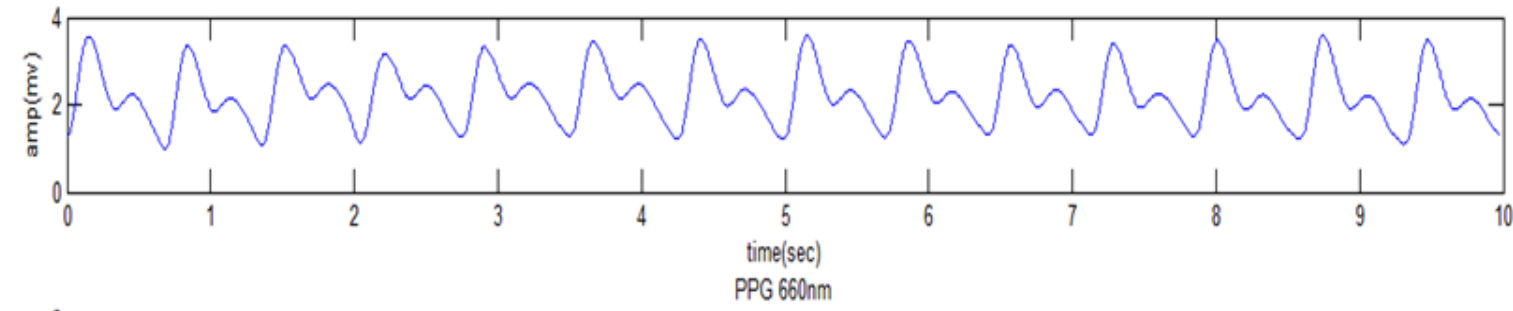

C)

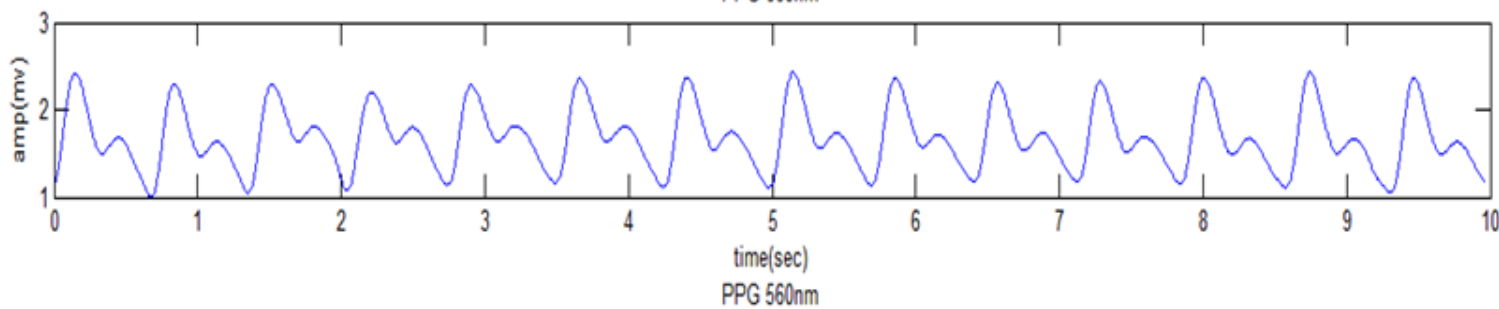

D)

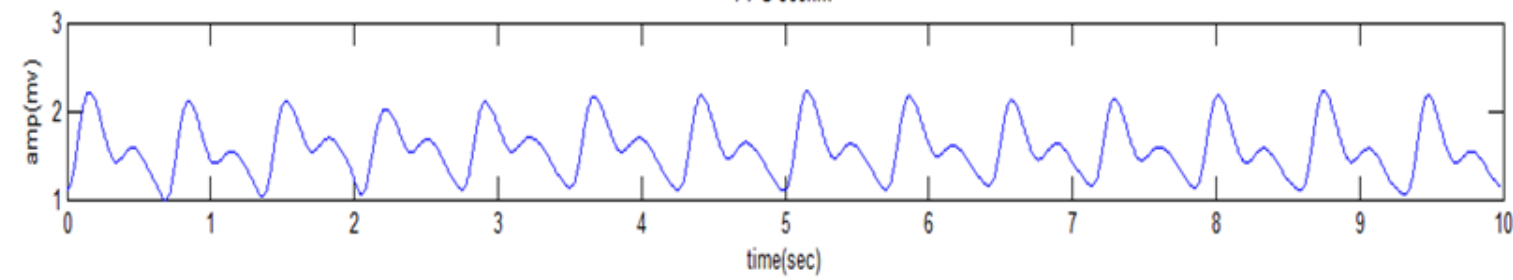

Figure 3: A) An example of recorded photoplethysmographic signal of a subject and separating the example at three wavelengths in order, B) $850 \mathrm{~nm}$, C) $660 \mathrm{~nm}, \&$ D) $560 \mathrm{~nm}$

In addition, the mean and standard deviation of the data were $43.43 \pm 3.43$. In Figure 5, the output of the HCT estimation function proportional to equation 4 for each subject is shown in Figure 2 with the same order. For the final estimation of the blood 
HCT percentage, the results of measurements with a standard pulse oximetry system were compared, and the calibration coefficient of equation four was determined based on the statistical population results. Accordingly, the mean HCT percentage obtained by the optic method was $43.31 \pm 33.37$.

Comparison of the final HCT level obtained by the proposed optical method with the reference values extracted from the standard laboratory technique showed a mean error of $0.6 \pm 0.25 \%$ in two groups. Analysis of correlation between laboratory data and photoplethysmography signal using Kendall's, Spearman's, and Pearson methods showed that the process of changes was significant in all three methods $(\mathrm{P}<0.01)$ and there was a positive correlation in this regard (Table 1).

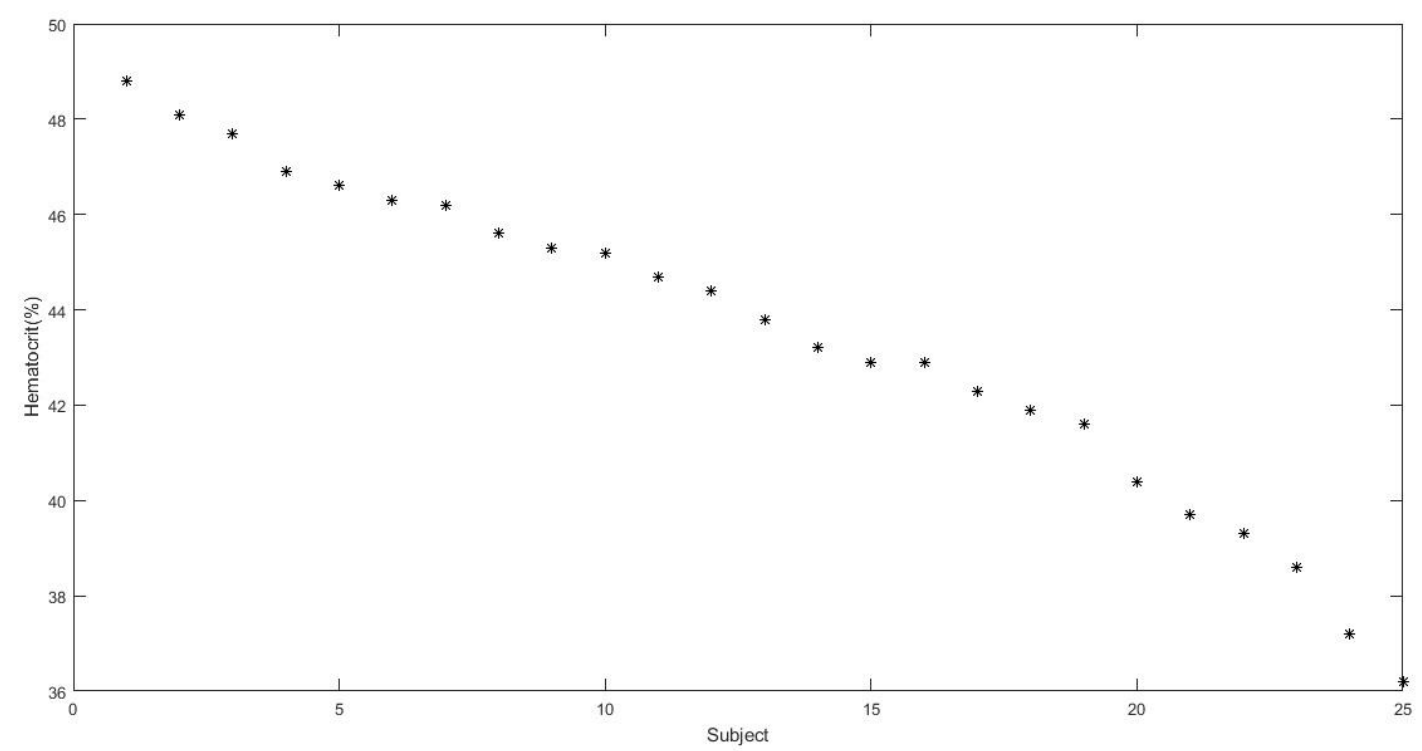

Figure 4: Illustration of results of blood HCT percentage in all subjects using blood test in a downward trend

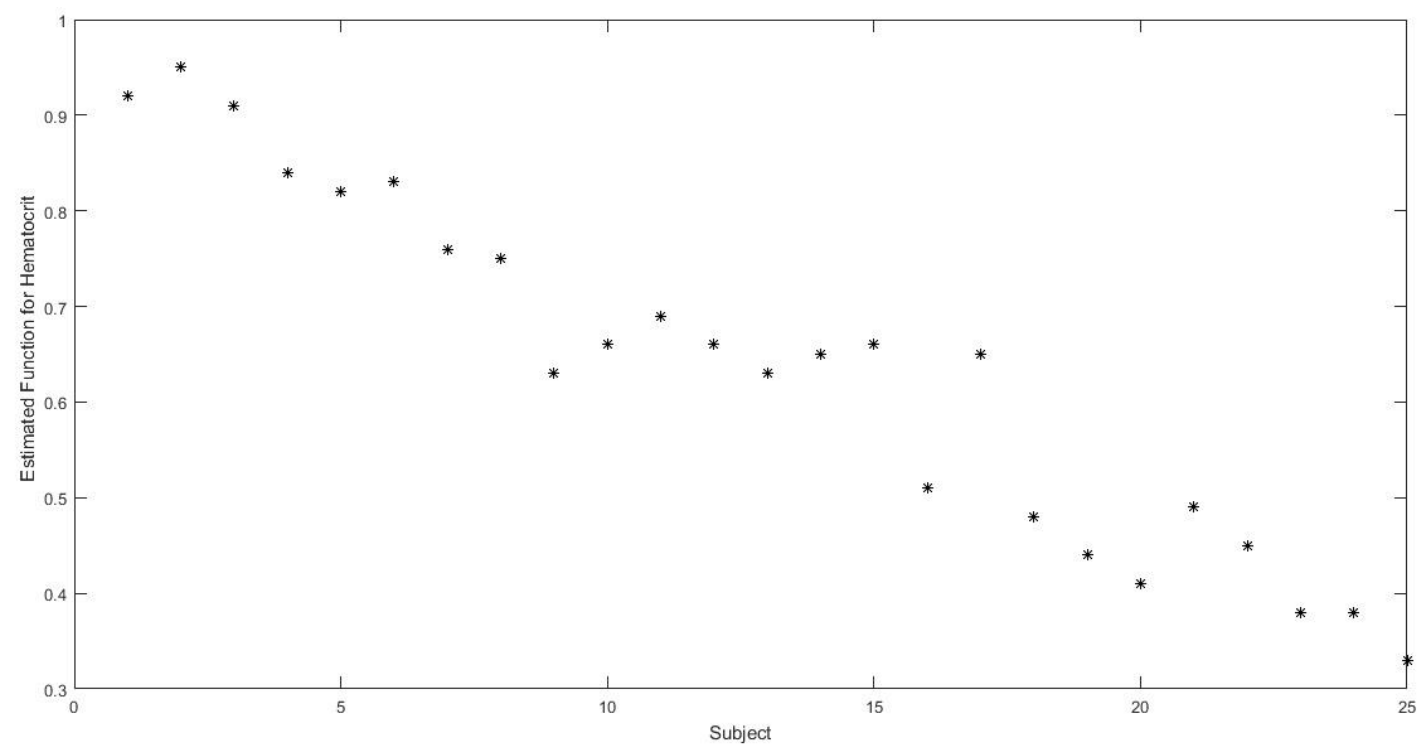

Figure 5: Results of blood HCT estimation function in all subjects using optical method introduced in the research 
Table 1: Evaluation of level of correlation between the laboratory data of blood HCT and optical signal

\begin{tabular}{llll}
\hline Evaluation method & Number of samples & Level of correlation & Level of significance \\
\hline Pearson & 25 & 0.949 & 0.01 \\
\hline Kendall & 25 & 0.860 & 0.01 \\
\hline Spearman & 25 & 0.955 & 0.01
\end{tabular}

\section{Discussion}

Considering the importance and applicability of the continuous diagnosis and monitoring of human blood components by optical and noninvasive methods, numerous studies have been performed in this area, and even some studies have evaluated the efficiency of techniques in special measurement conditions $(9,28)$. The amount of light absorption in the blood in the visible and near-infrared wavelength depends on hemoglobin and its derivatives, and the plasma of blood, which is basically water, $(11,12,15)$. Pulse changes in blood volume in the tissue are carried out by light irradiation and reflection into the tissue, which is known as photoplethysmography. The peak-to-peak ratio of Photoplethysmographic pulse amplitude at various wavelengths depends on light absorbance in human blood, from which we can extract proper information about the human blood components. The absorption coefficient of light in the visible and infrared range is dependent on hemoglobin and its derivatives and plasma. The absorption and diffusion of HCT are also affected by total hemoglobin concentration $(16,17)$.

Moreover, light reflection and dispersion depend on the wavelength, red blood cell hemoglobin, and oxygen level in the bloodstream. On the other hand, artifacts and deformation of red blood cells depend on the light transmission rate. However, artifacts might be related to Pulsatile blood flow. Other errors in the data include the abnormal hemoglobin concentration and some relevant diseases, in a way that one wavelength (5-8) or two wavelengths $(2,10,21)$ have been used for photoplethysmography and HCT detection in some studies. Considering the properties of various wavelengths in this study, we applied three wavelengths of nearinfrared $(850 \mathrm{~nm})$, red $(660 \mathrm{~mm})$ and green $(560 \mathrm{~nm})$ in order to eliminate artifacts and establish a suitable calibration. In this regard, the near-infrared wavelength was selected in a way that it jointly affected the calibration and HCT measurement and simplified the design and implementation processes.

The results of the optical method presented in this research were indicative of a higher correlation between the characteristics of the recorded photoplethysmographic signals and HCT whether we use one or two wavelengths, compared to previous studies $(3,5)$. Moreover, in the proposed method, there was a lower error rate in measurements, compared to the other studies that reported errors, which demonstrated the ability of the technique to play an effective role in this respect $(2,3,5$ 8). The preliminary results of this study confirmed that in the continuous measurement of HCT, the optical method with three wavelengths could facilitate the elimination of artifacts during signal recording and calibrate the system with higher precision. Compared 
to other studies, the need for multiple hardware and software processes for the elimination of interventional factors decreases in this technique (18-20). In addition to the

\section{Acknowledgements}

Hereby, we extend our gratitude to the Islamic Azad University of Mashhad for supporting the implementation of the research in the form of an independent research project with the code of 373 . In addition, we would like to thank the department of medical engineering and manager and personnel of laboratories of Bentolhoda and Aria Hospital, who assisted us in recording the laboratory samples.

\section{References}

1. Jedrzejewska-Szczerska M and Gnyba M. Optical investigation of hematocrit level in human blood. Optical and Acoustical Methods in Science and Technology. 2011;120.

2. Iftimia NV, Hammer DX, Bigelow CE. Toward noninvasive measurement of blood hematocrit using spectral domain low coherence interferometry and retinal tracking. Optical Society of America. 2006;14(8).

3. Xu X, Chen Z. Evaluation of hematocrit measurement using spectral domain optical coherence tomography. Proc. International Conference on BioMedical Engineering and Informatics Sanya. 2008;615-8.

4. Secomsky W, Nowicki A, Guidi F, Tortoli P, and Lewin PA. Non-invasive measurement of blood hematocrit in artery. Bulletin of the Polish Academy of Sciences. 2005;53(3):245-50.

5. Enejder A, Koo T, Oh J, Hunter M, Sasic S, Feld M. Blood analysis by Raman spectroscopy, Optics Lett. 2002;27:2004-6.

6. Sakudo A, Kato YH, Kuratsune H, Ikuta K. Non-invasive prediction of hematocrit levels by issues mentioned, low processing volume and high speed computing can be a good basis for the production and practical use of the suggested sensor.

\section{Declarations}

\section{Conflict of interest}

We declare that we have no financial or non-financial conflicts of interest related to the subject matter or materials discussed in the article.

\section{Authors' contributions}

All authors contributed equally to this work.

portable visible and near-infrared spectrophotometer. Clinica Chimica Acta. 2009; 408:123-7.

7. Schmitt JM, Zhou X, Miller J. Measurement of blood hematocrit by dual-wavelength near-IR photoplethysmography. SPIE Vol 1641. 1992; 150-61.

8. Timm U, Lewis E, McGrath D, Kraitl J, Ewald H. LED Based Sensor System for Non-Invasive Measurement of the Hemoglobin Concentration in Human Blood. IFMBE Proceedings. 2008;23:8258.

9. Timm U, Lewis E, McGrath D, Kraitl J, Ewald H. Sensor System Concept for Non-Invasive Blood Diagnosis. Procedia Chemistry 1. 2009;493-6.

10. Aldrich TK, Moosikasuwan M, Shah SD, Deshpande KS. Length-normalized pulse photoplethysmography: a noninvasive method to measure blood hemoglobin. Ann Biomed Eng. 2002;30:1291-8.

11. Jeon KJ, Kim SJ, Park KK, Kim JW, Yoon G. Noninvasive total hemoglobin measurement. J Biomed Opt. 2002;7:45-50. 
12. Doshi R, Panditrao A. Non-Invasive Optical Sensor for Hemoglobin Determination.

International Journal of Engineering Research and Applications (IJERA). 2013;3:559-62.

13. Kraitl J, Ewald H and Gehring H. An optical device to measure blood components by a photoplethysmographic method. J. Opt. A: Pure Appl. Opt. 2005;7:S318-24.

\section{Kraitl J, Ewald H. Results of hemoglobin} concentration measurements in whole blood with an optical non-invasive method. Photon, Optics and Photonics, IOP Conference. 2008.

15. US6606509B2. Method and apparatus for improving the accuracy of noninvasive hematocrit measurements. Schmitt JM. 2001.

16. US7011631. Noninvasive method of measuring blood density and hematocrit. Hemonix Inc. 2006.
17. US6662031. Method and device for the noninvasive determination of hemoglobin and hematocrit. Abbott Laboratories. 2003.

18. US6304767. Non-invasive optical measurement of blood hematocrit. Polestar Technologies. 2001.

19. Krystian E. Spectroscopic and wireless sensor of hematocrit level. Procedia Engineering. 2012;47:156-9.

20. Hiraokatq M, Firbankt M, Essenpreist M, Copet M, Anidget SR, Zees P, Delpyt DT. A Monte Carlo investigation of optical path length in inhomogeneous tissue and its application to near-infrared spectroscopy. Phys. Med. Biol. 1993;38:1859-76.

\section{How to cite:}

Azarnoosh M, Doostdar Noghabi H. Increasing the Accuracy of Blood Hematocrit Measurement by Triplicate Wavelength Photoplethysmography Method. Jorjani Biomedicine Journal. 2018; 6(4): 19-28. 